\title{
Inversion of Meg Data for a 2-D Current Distribution
}

\author{
George Dassios, Konstantia Satrazemi \\ Department of Chemical Engineering, University of Patras and ICE/HT-FORTH, Patras, Greece \\ Email: gdassios@otenet.gr
}

Received 4 May 2014; revised 2 June 2014; accepted 12 June 2014

Copyright (C) 2014 by authors and Scientific Research Publishing Inc. This work is licensed under the Creative Commons Attribution International License (CC BY). http://creativecommons.org/licenses/by/4.0/

(c) (7) Open Access

\begin{abstract}
The support of a localized three-dimensional neuronal current distribution, within a conducting medium, is not identifiable from knowledge of the exterior magnetic flux density, obtained via Magnetoencephalographic (MEG) measurements. However, this is not true if the neuronal current is supported on a set with dimensionality less than three. That is, the support of a dipolar current distribution can be recovered if it is a set of isolated points, a segment of a curve, or a surface patch. In this work we provide an analytic algorithm for this inverse MEG problem and apply it to the case where the current is supported on a localized disk having arbitrary position and size within the brain tissue. The proposed recovery algorithm reduces the identification of the characteristics of the current to the solution of a nonlinear algebraic system, which can be handled numerically.
\end{abstract}

\section{Keywords}

\section{Magnetoencephalography, Inversion of Current}

\section{Introduction}

Magnetoencephalography associates a neuronal current within the functional brain with the magnetic flux density and it creates outside the head. In particular, the direct problem consists of the calculation of the exterior magnetic field when the primary neuronal current is given, and the inverse problem seeks to identify the current that generates a given exterior magnetic field. The main difficulty with the inverse problem of Magnetoencephalography is due to the fact that, besides the excitation of the neuronal current within the conductive brain tissue, a secondary induction current is generated which, in a sense, makes the primary neuronal current less "visible" by the exterior magnetic field. The induction current is supported on the conductive brain tissue and therefore it depends on the geometry of the brain-head system. Even for relatively simple geometrical models such as 
a triaxial ellipsoid the calculations are very complicated [1]. An excellent review of the electromagnetic activity of the human brain can be found in [2], while the standard book for an introduction of the brain imaging modalities of Electroencephalography and Magnetoencephalography is the book by Malmivuo and Plonsey [3].

The most important question for the inverse problem is the question of uniqueness, that is, whether it is possible to recover the complete information about the current from the exterior magnetic data. The answer to this question is negative. The fact that a current within a conductor cannot be identified, from measurements of the exterior magnetic field it generates, was known to Helmholtz 160 years ago [4]. However, the problem of determining exactly what part of the current can be recovered was a topic of intense investigation during the last decade, and the final answer was given in [5]. A complete discussion of all the existing results on the topic can be found in [6]. Today we know that, no matter what the geometry of the brain-head system is, complete knowledge of the electric potential on the surface of the head can give us no more than one third of the neuronal current, and complete knowledge of the exterior magnetic field can give us no more than two thirds of the neuronal current, one of which is the one obtained from the electroencephalographic measurements. Hence, even in the very difficult situation where we have complete and synchronous data from both electroencephalographic and Magnetoencephalographic data, still one third of the current cannot be identified.

The next question one can ask, in connection to the inverse problem of Magnetoencephalography, has to do with the possibility to identify the position and the extent of a localized current. Albanese and Monk [7], have shown that this is not possible if the support of the current is a three-dimensional set. However, this is possible if the support of the current is a two-, one-, or zero-dimensional set. That is we can identify a localized current if it is supported on a set of isolated points, on a segment of a curve, or on a surface patch.

The purpose of this work is to provide a concrete example of the Albanese-Monk result. In particular, we consider a neuronal current that is supported on a small circular disk, orthogonal to the radius that passes through its center, and we construct an algebraic algorithm that solves the inverse Magnetoencephalographic problem. In fact, the identification of the location and the size of the supporting disk is reduced to the solution of a nonlinear algebraic system which can be solved numerically.

The paper is organized as follows. The solution to the problem of calculating the exterior magnetic potential for a single dipolar current within a conductive sphere is briefly described in Section 2 . This solution plays the role of the fundamental solution for the problem of Magnetoencephalography in spherical geometry. Section 3 contains the calculations that lead to the magnetic potential in the case where the source current is distributed on a small disk. Finally, Section 4 develops the algorithm for the inversion of the measured data in the case described in the previous section. An Appendix at the end of the paper provides some explanations about the long and tedious calculations that are used in Section 3.

\section{The MEG Problem for a Single Dipole}

Quasi-Static theory of Electromagnetism Magnetoencephalography [8]-[10] assumes that the time derivatives of the electric and magnetic fields are very small and therefore the corresponding terms in Maxwell's equations can be neglected. Then, it can be shown that the magnetic field, generated by a dipolar current at the point $\boldsymbol{r}_{0}$ having moment $\boldsymbol{Q}$, is given by the Geselowitz formula [11]

$$
\boldsymbol{B}\left(\boldsymbol{r} ; \boldsymbol{r}_{0}\right)=\frac{\mu_{0}}{4 \pi} \boldsymbol{Q} \times \frac{\boldsymbol{r}-\boldsymbol{r}_{0}}{\left|\boldsymbol{r}-\boldsymbol{r}_{0}\right|^{3}}-\frac{\mu_{0} \sigma}{4 \pi} \oint_{S} u\left(\boldsymbol{r}^{\prime} ; \boldsymbol{r}_{0}\right) \hat{\boldsymbol{n}}\left(\boldsymbol{r}^{\prime}\right) \times \frac{\boldsymbol{r}-\boldsymbol{r}^{\prime}}{\left|\boldsymbol{r}-\boldsymbol{r}^{\prime}\right|^{3}} \mathrm{~d} s\left(\boldsymbol{r}^{\prime}\right), \boldsymbol{r} \in \Omega^{c}
$$

where $u$ is the electric potential on the boundary $S$ of the conducting medium $\Omega$ representing the brainhead system. In Formula (1), $\Omega \Omega^{c}$ denotes the exterior domain, $\sigma$ is the constant conductivity of the brain tissue, $\mu_{0}$ is the common magnetic permeability both inside and outside $\Omega$ and $\hat{\boldsymbol{n}}$ stands for the outward unit normal on the boundary $S$.

If $\Omega$ is a sphere of radius $a$, then we know from the solution of the corresponding Electroencephalography problem [12], that the electric potential on the boundary of the sphere is given by

$$
u\left(\boldsymbol{r}^{\prime} ; \boldsymbol{r}_{0}\right)=\frac{1}{4 \pi \sigma}\left(\boldsymbol{Q} \cdot \nabla_{\boldsymbol{r}_{0}}\right) \sum_{n=1}^{\infty} \frac{2 n+1}{n} \frac{r_{0}^{n}}{\alpha^{n+1}} P_{n}\left(\hat{\boldsymbol{r}}^{\prime} \cdot \hat{\boldsymbol{r}}_{0}\right)=\frac{1}{\sigma}\left(\boldsymbol{Q} \cdot \nabla_{\boldsymbol{r}_{0}}\right) \sum_{n=1}^{\infty} \sum_{m=-n}^{n} \frac{1}{n} \frac{r_{0}^{n}}{\alpha^{n+1}} Y_{n}^{m}(\hat{\boldsymbol{r}}) Y_{n}^{m}\left(\hat{\boldsymbol{r}}^{\prime}\right)^{*}
$$

where $Y_{n}^{m}$ stands for the normalized complex spherical harmonics 


$$
Y_{n}^{m}(\hat{\boldsymbol{r}})=\sqrt{\frac{2 n+1}{4 \pi} \frac{(n-|m|) !}{(n+|m|) !}} P_{n}^{|m|}(\cos \vartheta) \mathrm{e}^{i m \phi}
$$

and $P_{n}^{m}$ denotes the Legendre functions of the first kind.

Inserting expression (2) in the Formula (1) and performing the indicated integration we obtain the magnetic field outside the sphere. However, since the magnetic field $\boldsymbol{B}$ in the exterior to the sphere is both solenoidal and irrotational it follows that there exists a scalar magnetic potential $U$, which is also harmonic, such that [10]

$$
\boldsymbol{B}\left(\boldsymbol{r} ; \boldsymbol{r}_{0}\right)=\nabla U\left(\boldsymbol{r} ; \boldsymbol{r}_{0}\right), r>a \text {. }
$$

Then, utilizing the orthogonality properties of the spherical harmonics we can calculate the magnetic potential and arrive at the following expression [12] [13]

$$
U\left(\boldsymbol{r} ; \boldsymbol{r}_{0}\right)=\mu_{0}\left(\boldsymbol{Q} \times \boldsymbol{r}_{0} \cdot \nabla_{\boldsymbol{r}_{0}}\right) \sum_{n=1}^{\infty} \sum_{m=-n}^{n} \frac{1}{(n+1)(2 n+1)} \frac{r_{0}^{n}}{r^{n+1}} Y_{n}^{m}(\hat{\boldsymbol{r}}) Y_{n}^{m}\left(\hat{\boldsymbol{r}}_{0}\right)^{*}=\frac{\mu_{0}}{4 \pi}\left(\boldsymbol{Q} \times \boldsymbol{r}_{0} \cdot \nabla_{\boldsymbol{r}_{0}}\right) \sum_{n=1}^{\infty} \frac{1}{n+1} \frac{r_{0}^{n}}{r^{n+1}} P_{n}\left(\hat{\boldsymbol{r}} \cdot \hat{\boldsymbol{r}_{0}}\right) .
$$

The above expression provides the magnetic potential in the exterior of the sphere due to a single current dipole $\left\{\boldsymbol{r}_{0}, \boldsymbol{Q}\right\}$. Therefore, it can be considered as the fundamental solution of the MEG problem for the spherical geometry [14]. Consequently, any discrete, or continuous, current distribution can be obtained through summation, or integration, respectively, of the above fundamental solution [15]. Then, Formula (3) gives the exterior magnetic field.

\section{The Field of a 2-D Current Distribution}

Suppose now that the neuronal current $\boldsymbol{J}^{p}$ is supported on a small part of a smooth surface which is located around a central point $\boldsymbol{r}_{0}$. Let this little surface be represented by the equation

$$
\boldsymbol{r}=\boldsymbol{r}(s, t),(s, t) \in\left[s_{1}, s_{2}\right] \times\left[t_{1}, t_{2}\right], \quad \boldsymbol{r}\left(s_{0}, t_{0}\right)=\boldsymbol{r}_{0}
$$

where $r_{0}$ is some interior point of this surface. Then the current is described by the function

$$
\boldsymbol{J}^{p}=\boldsymbol{J}^{p}(\boldsymbol{r}(s, t)),(s, t) \in\left[-s_{0}, s_{0}\right] \times\left[-t_{0}, t_{0}\right]
$$

which, if it is assumed to be small, it can be represented by the linear part of its Taylor expansion

$$
\begin{aligned}
\boldsymbol{J}^{p}(\boldsymbol{r}(s, t)) & \approx \boldsymbol{J}^{p}\left(\boldsymbol{r}\left(s_{0}, t_{0}\right)\right)+\left.\left(s-s_{0}\right) \frac{\partial \boldsymbol{J}^{p}(\boldsymbol{r}(s, t))}{\partial s}\right|_{\left(s_{0}, t_{0}\right)}+\left.\left(t-t_{0}\right) \frac{\partial \boldsymbol{J}^{p}(\boldsymbol{r}(s, t))}{\partial t}\right|_{\left(s_{0}, t_{0}\right)} \\
& =\boldsymbol{J}^{p}\left(\boldsymbol{r}_{0}\right)+\left(s-s_{0}\right) \frac{\partial \boldsymbol{J}^{p}\left(\boldsymbol{r}_{0}\right)}{\partial s}+\left(t-t_{0}\right) \frac{\partial \boldsymbol{J}^{p}\left(\boldsymbol{r}_{0}\right)}{\partial t} .
\end{aligned}
$$

In particular, if the surface that curies the current is a small disk of radius $\varepsilon$, which is centered at $\boldsymbol{r}_{0}$ and has the vector $\boldsymbol{r}_{0}$ perpendicular to its plane, then, this disk is defined by

$$
\left(\boldsymbol{r}-\boldsymbol{r}_{0}\right) \cdot \boldsymbol{r}_{0}=0,\left|\boldsymbol{r}-\boldsymbol{r}_{0}\right| \leq \varepsilon .
$$

Utilizing the freedom we have to select a coordinate system we choose the direction $\hat{\boldsymbol{r}}_{0}$ to coincide with the $x_{3}$-axis. Then a parametric representation of the circle is given by

$$
\boldsymbol{r}=x_{1} \hat{\boldsymbol{x}}_{1}+x_{2} \hat{\boldsymbol{x}}_{2}+r_{0} \hat{\boldsymbol{x}}_{3}, x_{1}^{2}+x_{2}^{2} \leq \varepsilon^{2}
$$

and $\boldsymbol{r}_{0}=r_{0} \hat{\boldsymbol{x}}_{3}$. In this case, the third Cartesian coordinate of $\boldsymbol{r}$ is always equal to $r_{0}$, and the linear Taylor approximation assumes the form

$$
\begin{aligned}
\boldsymbol{J}^{p}(\boldsymbol{r}) & \approx \boldsymbol{J}^{p}\left(\boldsymbol{r}_{0}\right)+\left(x_{1}-0\right) \frac{\partial \boldsymbol{J}^{p}\left(\boldsymbol{r}_{0}\right)}{\partial x_{1}}+\left(x_{2}-0\right) \frac{\partial \boldsymbol{J}^{p}\left(\boldsymbol{r}_{0}\right)}{\partial x_{2}}+\left(x_{3}-r_{0}\right) \frac{\partial \boldsymbol{J}^{p}\left(\boldsymbol{r}_{0}\right)}{\partial x_{2}} \\
& =\boldsymbol{J}^{p}\left(\boldsymbol{r}_{0}\right)+x_{1} \frac{\partial \boldsymbol{J}^{p}\left(\boldsymbol{r}_{0}\right)}{\partial x_{1}}+x_{2} \frac{\partial \boldsymbol{J}^{p}\left(\boldsymbol{r}_{0}\right)}{\partial x_{2}}=\boldsymbol{Q}+x_{1} \boldsymbol{D}^{1}+x_{2} \boldsymbol{D}^{2}
\end{aligned}
$$

where $\boldsymbol{D}^{1}, \boldsymbol{D}^{2}$ denote the constant vectors 


$$
\boldsymbol{D}^{i}=\left(D_{1}^{i}, D_{2}^{i}, D_{3}^{i}\right)=\frac{\partial \boldsymbol{J}^{p}\left(\boldsymbol{r}_{0}\right)}{\partial x_{i}}, \quad i=1,2
$$

and $\boldsymbol{Q}=\left(Q_{1}, Q_{2}, Q_{3}\right)=\boldsymbol{J}^{p}\left(\boldsymbol{r}_{0}\right)$ denotes the average moment over the small circle. In order to interpret Formula (11) in an invariant form, we introduce a third vector $\boldsymbol{D}^{3}=\left(D_{1}^{3}, D_{2}^{3}, D_{3}^{3}\right)$, which is also constant, as well as the dyadic [16]

$$
\tilde{\boldsymbol{D}}=\sum_{i=1}^{3} \sum_{j=1}^{3} D_{j}^{i} \hat{\boldsymbol{x}}_{i} \otimes \hat{\boldsymbol{x}}_{j}
$$

Then, the absence of a term involving $x_{3}$ in Formula (11) can be interpreted as the constrain

$$
\hat{\boldsymbol{x}}_{3} \cdot \tilde{\boldsymbol{D}}=\left(D_{1}^{3}, D_{2}^{3}, D_{3}^{3}\right)=(0,0,0)
$$

which means that, the left contraction of the dyadic $\tilde{\boldsymbol{D}}$ with the direction normal to the disk vanishes. Consequently, in the general case of an arbitrary oriented system, we should have the condition

$$
\hat{\boldsymbol{r}}_{0} \cdot \tilde{\boldsymbol{D}}=(0,0,0)
$$

which gives the three scalar conditions

$$
\begin{aligned}
& x_{01} D_{1}^{1}+x_{02} D_{1}^{2}+x_{03} D_{1}^{3}=0 \\
& x_{01} D_{2}^{1}+x_{02} D_{2}^{2}+x_{03} D_{2}^{3}=0 \\
& x_{01} D_{3}^{1}+x_{02} D_{3}^{2}+x_{03} D_{3}^{3}=0 .
\end{aligned}
$$

Consequently, the approximate current (11) is now written as

$$
\boldsymbol{J}^{p}(\boldsymbol{r}) \approx \boldsymbol{Q}+\boldsymbol{r} \cdot \tilde{\boldsymbol{D}} \text {. }
$$

We can utilize the coordinate invariance principle to simplify our calculations. We actually want to calculate the total magnetic potential, given in (5), which is generated by the approximate current (28). Since our ultimate goal is to invert the MEG data that will give us the quantities $\boldsymbol{Q}, \boldsymbol{r}_{0}$ and $\varepsilon$ we will calculate as many terms of the expansion (5) as we actually need.

For the generic excitation dipole $\left\{\boldsymbol{r}^{\prime}, \boldsymbol{J}\left(\boldsymbol{r}^{\prime}\right)\right\}$, Formula (5) is written as

$$
\begin{aligned}
U\left(\boldsymbol{r} ; \boldsymbol{r}^{\prime}\right)= & \frac{\mu_{0}}{4 \pi \sigma}\left(\boldsymbol{J}^{p}\left(\boldsymbol{r}^{\prime}\right) \times \boldsymbol{r}^{\prime}\right) \cdot\left[\frac{1}{2 r^{2}} \nabla_{\boldsymbol{r}^{\prime}}\left(r^{\prime} P_{1}\left(\hat{\boldsymbol{r}} \cdot \hat{\boldsymbol{r}}^{\prime}\right)\right)+\frac{1}{3 r^{3}} \nabla_{\boldsymbol{r}^{\prime}}\left(r^{\prime 2} P_{2}\left(\hat{\boldsymbol{r}} \cdot \hat{\boldsymbol{r}}^{\prime}\right)\right)\right. \\
& \left.+\frac{1}{4 r^{4}} \nabla_{\boldsymbol{r}^{\prime}}\left(r^{\prime 3} P_{3}\left(\hat{\boldsymbol{r}} \cdot \hat{\boldsymbol{r}}^{\prime}\right)\right)\right]+O\left(r^{-5}\right)
\end{aligned}
$$

and through some direct calculation with the Legend polynomials [17] we obtain the following relations, which are written in dyadic form [16] in order to isolate the factors that are going to be integrated

$$
\begin{array}{r}
\nabla_{\boldsymbol{r}^{\prime}}\left(r^{\prime} P_{1}\left(\hat{\boldsymbol{r}} \cdot \hat{\boldsymbol{r}}^{\prime}\right)\right)=\hat{\boldsymbol{r}} \\
\nabla_{\boldsymbol{r}^{\prime}}\left(r^{\prime 2} P_{2}\left(\hat{\boldsymbol{r}} \cdot \hat{\boldsymbol{r}}^{\prime}\right)\right)=(3 \hat{\boldsymbol{r}} \otimes \hat{\boldsymbol{r}}-\tilde{\boldsymbol{I}}) \cdot \boldsymbol{r}^{\prime} \\
\nabla_{\boldsymbol{r}^{\prime}}\left(r^{\prime 3} P_{3}\left(\hat{\boldsymbol{r}} \cdot \hat{\boldsymbol{r}}^{\prime}\right)\right)=\frac{3}{2}(5 \hat{\boldsymbol{r}} \otimes \hat{\boldsymbol{r}} \otimes \hat{\boldsymbol{r}}-2 \tilde{\boldsymbol{I}} \otimes \hat{\boldsymbol{r}}-\hat{\boldsymbol{r}} \otimes \tilde{\boldsymbol{I}}): \boldsymbol{r}^{\prime} \otimes \boldsymbol{r}^{\prime}
\end{array}
$$

The symbol $\tilde{\boldsymbol{I}}$ denotes the identity dyadic, “:” defines the double contraction [16]

$$
(\boldsymbol{a} \otimes \boldsymbol{b}):(\boldsymbol{c} \otimes \boldsymbol{d})=(\boldsymbol{b} \cdot \boldsymbol{c})(\boldsymbol{a} \cdot \boldsymbol{d})
$$

and similarly the triple contraction [16] is defined as

$$
(\boldsymbol{a} \otimes \boldsymbol{b} \otimes \boldsymbol{c}):(\boldsymbol{d} \otimes \boldsymbol{e} \otimes \boldsymbol{f})=(\boldsymbol{c} \cdot \boldsymbol{d})(\boldsymbol{b} \cdot \boldsymbol{e})(\boldsymbol{a} \cdot \boldsymbol{f}) .
$$

Furthermore, the exterior magnetic potential given in (29) can be written in its Cartesian form [13], [15] as follows 


$$
U\left(\boldsymbol{r} ; \boldsymbol{r}^{\prime}\right)=\frac{\mu_{0}}{4 \pi}\left[\frac{H_{1}(\boldsymbol{r})}{r^{3}}+\frac{H_{2}(\boldsymbol{r})}{r^{5}}+\frac{H_{3}(\boldsymbol{r})}{r^{7}}\right]+O\left(r^{-5}\right)
$$

where the coefficients

$$
\begin{gathered}
H_{1}(\boldsymbol{r})=\frac{1}{2} \boldsymbol{r} \cdot\left(\boldsymbol{J}^{p}\left(\boldsymbol{r}^{\prime}\right) \times \boldsymbol{r}^{\prime}\right) \\
H_{2}(\boldsymbol{r})=\boldsymbol{r} \otimes \boldsymbol{r}: \boldsymbol{r}^{\prime} \otimes\left(\boldsymbol{J}^{p}\left(\boldsymbol{r}^{\prime}\right) \times \boldsymbol{r}^{\prime}\right) \\
H_{3}(\boldsymbol{r})=\frac{3}{8}\left(5 \boldsymbol{r} \otimes \boldsymbol{r} \otimes \boldsymbol{r}-\boldsymbol{r}^{2} \boldsymbol{r} \otimes \tilde{\boldsymbol{I}}\right) ! \boldsymbol{r}^{\prime} \otimes \boldsymbol{r}^{\prime} \otimes\left(\boldsymbol{J}^{p}\left(\boldsymbol{r}^{\prime}\right) \times \boldsymbol{r}^{\prime}\right)
\end{gathered}
$$

are homogeneous harmonic functions [15].

In order to obtain the magnetic potential, generated by the neuronal current which is distributed over the disk $D_{\varepsilon}$ of radius $\varepsilon$, we need to substitute the expressions (19) in (27), (28) and (29) and integrate the resulting equations over the disk $D_{\varepsilon}$. We perform the calculations in the special case where $\hat{\boldsymbol{r}}_{0}=\hat{\boldsymbol{x}}_{3}$ and express the results in terms of $\hat{\boldsymbol{x}}_{3}$. Then, in the resulting expressions we replace $\hat{\boldsymbol{x}}_{3}$ by the arbitrary vector $\boldsymbol{r}_{0}$ to obtain the corresponding generic results. The final expressions are given in the sequel

$$
\begin{aligned}
& H_{1}(\boldsymbol{r})= \frac{1}{2} \boldsymbol{r} \cdot \oint_{D_{\varepsilon}}\left[\boldsymbol{Q} \times \boldsymbol{r}^{\prime}+\boldsymbol{r}^{\prime} \cdot\left(\tilde{\boldsymbol{D}} \times \boldsymbol{r}^{\prime}\right)\right] \mathrm{d} s\left(\boldsymbol{r}^{\prime}\right)=\frac{\pi \varepsilon^{2}}{8} \boldsymbol{r} \cdot\left(4 \boldsymbol{Q} \times \boldsymbol{r}_{0}-\varepsilon^{2} \boldsymbol{d}\right) \\
& H_{2}(\boldsymbol{r})=\boldsymbol{r} \otimes \boldsymbol{r}: \oint_{D_{\varepsilon}} \boldsymbol{r}^{\prime} \otimes\left[\boldsymbol{Q} \times \boldsymbol{r}^{\prime}+\boldsymbol{r}^{\prime} \cdot\left(\tilde{\boldsymbol{D}} \times \boldsymbol{r}^{\prime}\right)\right] \mathrm{d} s\left(\boldsymbol{r}^{\prime}\right)=\frac{\pi \varepsilon^{2}}{4 r_{0}^{2}}\left(\varepsilon^{2}-4 r_{0}^{2}\right)(\boldsymbol{r} \otimes \boldsymbol{r}):\left[\boldsymbol{r}_{0} \otimes\left(\boldsymbol{r}_{0} \times \boldsymbol{Q}\right)\right] \\
&-\frac{\pi \varepsilon^{4}}{4}\left[\left(\boldsymbol{r}_{0} \otimes \boldsymbol{r}\right):(\tilde{\boldsymbol{D}} \times \boldsymbol{r})+(\boldsymbol{r} \otimes \boldsymbol{r}):\left(\boldsymbol{r}_{0} \otimes \boldsymbol{d}\right)\right] \\
& H_{3}(\boldsymbol{r})= \frac{3}{8}\left(5 \boldsymbol{r} \otimes \boldsymbol{r} \otimes \boldsymbol{r}-r^{2} \boldsymbol{r} \otimes \tilde{\boldsymbol{I}}\right): \oint_{D_{\varepsilon}} \boldsymbol{r}^{\prime} \otimes \boldsymbol{r}^{\prime} \otimes\left[\boldsymbol{Q} \times \boldsymbol{r}^{\prime}+\boldsymbol{r}^{\prime} \cdot\left(\tilde{\boldsymbol{D}} \times \boldsymbol{r}^{\prime}\right)\right] \mathrm{d} s\left(\boldsymbol{r}^{\prime}\right) \\
&= \frac{3 \pi \varepsilon^{2}}{32 r_{0}^{2}}\left[r_{0}^{2} r^{2}\left(4 r_{0}^{2}-5 \varepsilon^{2}\right)-5\left(\boldsymbol{r} \cdot \boldsymbol{r}_{0}\right)^{2}\left(4 r_{0}^{2}-3 \varepsilon^{2}\right)\right]\left(\boldsymbol{r} \cdot \boldsymbol{r}_{0} \times \boldsymbol{Q}\right) \\
&+\frac{\pi \varepsilon^{4}}{64 r_{0}^{2}}\left(6 r_{0}^{2}-\varepsilon^{2}\right) \boldsymbol{r} \cdot\left[r_{0}^{2} r^{2} \boldsymbol{d}-5\left(\boldsymbol{r} \cdot \boldsymbol{r}_{0}\right)^{2} \boldsymbol{d}+10\left(\boldsymbol{r} \cdot \boldsymbol{r}_{0}\right)\left(\boldsymbol{r} \cdot \tilde{\boldsymbol{D}} \times \boldsymbol{r}_{0}\right)\right]
\end{aligned}
$$

where

$$
\boldsymbol{d}=\sum_{i=1}^{3} \sum_{i=1}^{3} D_{j}^{i}\left(\hat{\boldsymbol{x}}_{i} \times \hat{\boldsymbol{x}}_{j}\right)=\left(D_{3}^{2}-D_{2}^{3}\right) \hat{\boldsymbol{x}}_{1}+\left(D_{1}^{3}-D_{3}^{1}\right) \hat{\boldsymbol{x}}_{2}+\left(D_{2}^{1}-D_{1}^{2}\right) \hat{\boldsymbol{x}}_{3}
$$

is the vector invariant [16] of the dyadic $\tilde{\boldsymbol{D}}$.

The actual calculations that led to the above expressions, and especially to the Formula (32), are involved and quiet long. In order to facilitate the reader we provide, in the Appendix, an outline of the basic steps that lead the above expressions.

If we insert the above expressions of the harmonic functions $H_{1}, H_{2}, H_{3}$ in the expansion (26) we arrive at the Cartesian representation of the exterior magnetic potential $U$ up to the terms of order $r^{-5}$. That solves the forward MEG problem for a neuronal excitation that is supported on a small disk.

\section{Inversion of MEG Data}

In the previous section we defined the functions $H_{1}, H_{2}$ and $H_{3}$ which are homogeneous harmonic polynomials of degree 1,2 and 3 , respectively. These functions assume the forms

$$
\begin{gathered}
H_{1}(\boldsymbol{r})=A_{1} x_{1}+A_{2} x_{2}+A_{3} x_{3} \\
H_{2}(\boldsymbol{r})=B_{1} x_{1}^{2}+B_{2} x_{2}^{2}+B_{3} x_{3}^{2}+B_{12} x_{1} x_{2}+B_{23} X_{2} x_{3}+B_{31} x_{3} x_{1}
\end{gathered}
$$

with 


$$
B_{1}+B_{2}+B_{3}=0
$$

and

$$
\begin{aligned}
H_{3}(\boldsymbol{r})= & C_{1} x_{1}^{3}+C_{2} x_{2}^{3}+C_{3} x_{3}^{3}+C_{12} x_{1}^{2} x_{2}+C_{21} x_{2}^{2} x_{1}+C_{23} x_{2}^{2} x_{3}+C_{32} x_{3}^{2} x_{2} \\
& +C_{31} x_{3}^{2} x_{1}+C_{13} x_{1}^{2} x_{3}+C_{123} x_{1} x_{2} x_{3}
\end{aligned}
$$

with

$$
\begin{aligned}
& 3 C_{1}+C_{21}+C_{31}=0 \\
& C_{12}+3 C_{2}+C_{32}=0 \\
& C_{13}+C_{23}+3 C_{3}=0 .
\end{aligned}
$$

In the present work we assume the idealized case where the exterior magnetic potential $U$ is known. This means that expansion (26) is known and therefore the coefficients $A, B$ and $C$ are also known. Consequently, if we rewrite the polynomials $H_{1}, H_{2}$ and $H_{3}$ in terms of the Cartesian monomials that appear in (34), (35) and (37), then we can utilize their linear independence to equate each monomial with the corresponding known coefficient $A, B$ or $C$. In doing so for the first degree polynomial, we obtain from (30) and (34)

$$
\begin{aligned}
& A_{1}=\frac{\pi \varepsilon^{2}}{2}\left(Q_{2} x_{03}-Q_{3} x_{02}\right)-\frac{\pi \varepsilon^{4}}{8}\left(D_{3}^{2}-D_{2}^{3}\right) \\
& A_{2}=\frac{\pi \varepsilon^{2}}{2}\left(Q_{3} x_{01}-Q_{1} x_{03}\right)-\frac{\pi \varepsilon^{4}}{8}\left(D_{1}^{3}-D_{3}^{1}\right) \\
& A_{3}=\frac{\pi \varepsilon^{2}}{2}\left(Q_{1} x_{02}-Q_{2} x_{01}\right)-\frac{\pi \varepsilon^{4}}{8}\left(D_{2}^{1}-D_{1}^{2}\right)
\end{aligned}
$$

or

$$
\boldsymbol{A}=\left(A_{1}, A_{2}, A_{3}\right)=\frac{\pi \varepsilon^{2}}{8}\left(4 \boldsymbol{Q} \times \boldsymbol{r}_{0}-\varepsilon^{2} \boldsymbol{d}\right)
$$

where $\boldsymbol{d}$ is the vector invariant of the dyadic $\tilde{\boldsymbol{D}}$, defined by Equation (33). Equating the coefficients of the harmonic polynomials of the second degree, given in the Equations (31) and (35), we arrive at the relations

$$
\begin{aligned}
& B_{1}=\frac{1}{4} \pi \varepsilon^{2}\left[\left(4-\frac{\varepsilon^{2}}{r_{0}^{2}}\right)\left(Q_{2} x_{03}-Q_{3} x_{02}\right) x_{01}-\varepsilon^{2}\left(x_{01} D_{3}^{2}-x_{01} D_{2}^{3}+x_{02} D_{3}^{1}-x_{03} D_{2}^{1}\right)\right] \\
& B_{2}=\frac{1}{4} \pi \varepsilon^{2}\left[\left(4-\frac{\varepsilon^{2}}{r_{0}^{2}}\right)\left(Q_{3} x_{01}-Q_{1} x_{03}\right) x_{02}-\varepsilon^{2}\left(x_{02} D_{1}^{3}-x_{02} D_{3}^{1}+x_{03} D_{1}^{2}-x_{01} D_{3}^{2}\right)\right] \\
& B_{3}=\frac{1}{4} \pi \varepsilon^{2}\left[\left(4-\frac{\varepsilon^{2}}{r_{0}^{2}}\right)\left(Q_{1} x_{02}-Q_{2} x_{01}\right) x_{03}-\varepsilon^{2}\left(x_{03} D_{2}^{1}-x_{03} D_{1}^{2}+x_{01} D_{2}^{3}-x_{02} D_{1}^{3}\right)\right] \\
& B_{12}= \frac{1}{4} \pi \varepsilon^{2}\left(4-\frac{\varepsilon^{2}}{r_{0}^{2}}\right)\left[\left(Q_{3} x_{01}-Q_{1} x_{03}\right) x_{01}+\left(Q_{2} x_{03}-Q_{3} x_{02}\right) x_{02}\right] \\
&-\frac{1}{4} \pi \varepsilon^{4}\left[x_{01}\left(D_{1}^{3}-2 D_{3}^{1}\right)+x_{02}\left(2 D_{3}^{2}-D_{2}^{3}\right)+x_{03}\left(D_{1}^{1}-D_{2}^{2}\right)\right] \\
& B_{23}= \frac{1}{4} \pi \varepsilon^{2}\left(4-\frac{\varepsilon^{2}}{r_{0}^{2}}\right)\left[\left(Q_{1} x_{02}-Q_{2} x_{01}\right) x_{02}+\left(Q_{3} x_{01}-Q_{1} x_{03}\right) x_{03}\right] \\
&-\frac{1}{4} \pi \varepsilon^{4}\left[x_{02}\left(D_{2}^{1}-2 D_{1}^{2}\right)+x_{03}\left(2 D_{1}^{3}-D_{3}^{1}\right)+x_{01}\left(D_{2}^{2}-D_{3}^{3}\right)\right]
\end{aligned}
$$




$$
\begin{aligned}
B_{31}= & \frac{1}{4} \pi \varepsilon^{2}\left(4-\frac{\varepsilon^{2}}{r_{0}^{2}}\right)\left[\left(Q_{2} x_{03}-Q_{3} x_{02}\right) x_{03}+\left(Q_{1} x_{02}-Q_{2} x_{01}\right) x_{01}\right] \\
& -\frac{1}{4} \pi \varepsilon^{4}\left[x_{03}\left(D_{3}^{2}-2 D_{2}^{3}\right)+x_{01}\left(2 D_{2}^{1}-D_{1}^{2}\right)+x_{02}\left(D_{3}^{3}-D_{1}^{1}\right)\right]
\end{aligned}
$$

where the constrain (36) is easily verified.

Next we consider the cubic polynomials (32) and (37). The coefficients of the cubic monomials $x_{i}^{3}, i=1,2,3$ provide the equations

$$
\begin{aligned}
C_{1}= & \frac{3}{32} \pi \varepsilon^{2}\left(Q_{2} x_{03}-Q_{3} x_{02}\right)\left[5 \varepsilon^{2}\left(1-\frac{3 x_{01}^{2}}{r_{0}^{2}}\right)-4\left(r_{0}^{2}-5 x_{01}^{2}\right)\right] \\
& +\frac{3}{32} \pi \varepsilon^{4}\left(1-\frac{\varepsilon^{2}}{6 r_{0}^{2}}\right)\left[\left(D_{3}^{2}-D_{2}^{3}\right)\left(r_{0}^{2}-5 x_{01}^{2}\right)-10 x_{01}\left(x_{02} D_{3}^{1}-x_{03} D_{2}^{1}\right)\right] \\
C_{2}= & \frac{3}{32} \pi \varepsilon^{2}\left(Q_{3} x_{01}-Q_{1} x_{03}\right)\left[5 \varepsilon^{2}\left(1-\frac{3 x_{02}^{2}}{r_{0}^{2}}\right)-4\left(r_{0}^{2}-5 x_{02}^{2}\right)\right] \\
& +\frac{3}{32} \pi \varepsilon^{4}\left(1-\frac{\varepsilon^{2}}{6 r_{0}^{2}}\right)\left[\left(D_{1}^{3}-D_{3}^{1}\right)\left(r_{0}^{2}-5 x_{02}^{2}\right)-10 x_{02}\left(x_{03} D_{1}^{2}-x_{01} D_{3}^{2}\right)\right] \\
C_{3}= & \frac{3}{32} \pi \varepsilon^{2}\left(Q_{1} x_{02}-Q_{2} x_{01}\right)\left[5 \varepsilon^{2}\left(1-\frac{3 x_{03}^{2}}{r_{0}^{2}}\right)-4\left(r_{0}^{2}-5 x_{03}^{2}\right)\right] \\
+ & \frac{3}{32} \pi \varepsilon^{4}\left(1-\frac{\varepsilon^{2}}{6 r_{0}^{2}}\right)\left[\left(D_{2}^{1}-D_{1}^{2}\right)\left(r_{0}^{2}-5 x_{03}^{2}\right)-10 x_{03}\left(x_{01} D_{2}^{3}-x_{02} D_{1}^{3}\right)\right] .
\end{aligned}
$$

The coefficients of the monomials involving the cross-product terms $x_{i}^{2} x_{j}, i \neq j, i, j=1,2,3$, provide the following equations

$$
\begin{aligned}
C_{12}= & \frac{3}{32} \pi \varepsilon^{2}\left(Q_{3} x_{01}-Q_{1} x_{03}\right)\left[5 \varepsilon^{2}\left(1-\frac{3 x_{01}^{2}}{r_{0}^{2}}\right)-4\left(r_{0}^{2}-5 x_{01}^{2}\right)\right]+\frac{15}{16} \pi \varepsilon^{2}\left(Q_{2} x_{03}-Q_{3} x_{02}\right)\left(4-\frac{3 \varepsilon^{2}}{r_{0}^{2}}\right) x_{01} x_{02} \\
& +\frac{3}{32} \pi \varepsilon^{4}\left(1-\frac{\varepsilon^{2}}{6 r_{0}^{2}}\right)\left[\left(D_{1}^{3}-D_{3}^{1}\right)\left(r_{0}^{2}-5 x_{01}^{2}\right)-10\left(D_{3}^{2}-D_{2}^{3}\right) x_{01} x_{02}-10 x_{02}\left(x_{02} D_{3}^{1}-x_{03} D_{2}^{1}\right)\right. \\
& \left.-10 x_{01}\left(x_{03} D_{1}^{1}-x_{01} D_{3}^{1}\right)-10 x_{01}\left(x_{02} D_{3}^{2}-x_{03} D_{2}^{2}\right)\right] \\
C_{21}= & \frac{3}{32} \pi \varepsilon^{2}\left(Q_{2} x_{03}-Q_{3} x_{02}\right)\left[5 \varepsilon^{2}\left(1-\frac{3 x_{02}^{2}}{r_{0}^{2}}\right)-4\left(r_{0}^{2}-5 x_{02}^{2}\right)\right]+\frac{15}{16} \pi \varepsilon^{2}\left(Q_{3} x_{01}-Q_{1} x_{03}\right)\left(4-\frac{3 \varepsilon^{2}}{r_{0}^{2}}\right) x_{01} x_{02} \\
& -\frac{3}{32} \pi \varepsilon^{4}\left(1-\frac{\varepsilon^{2}}{6 r_{0}^{2}}\right)\left[\left(D_{2}^{3}-D_{3}^{2}\right)\left(r_{0}^{2}-5 x_{02}^{2}\right)-10\left(D_{3}^{1}-D_{1}^{3}\right) x_{01} x_{02}-10 x_{01}\left(x_{01} D_{3}^{2}-x_{03} D_{1}^{2}\right)\right. \\
& \left.-10 x_{02}\left(x_{03} D_{2}^{2}-x_{02} D_{3}^{2}\right)-10 x_{02}\left(x_{01} D_{3}^{1}-x_{03} D_{1}^{1}\right)\right] \\
C_{23}= & \frac{3}{32} \pi \varepsilon^{2}\left(Q_{1} x_{02}-Q_{2} x_{01}\right)\left[5 \varepsilon^{2}\left(1-\frac{3 x_{02}^{2}}{r_{0}^{2}}\right)-4\left(r_{0}^{2}-5 x_{02}^{2}\right)\right]+\frac{15}{16} \pi \varepsilon^{2}\left(Q_{3} x_{01}-Q_{1} x_{03}\right)\left(4-\frac{3 \varepsilon^{2}}{r_{0}^{2}}\right) x_{02} x_{03} \\
& +\frac{3}{32} \pi \varepsilon^{4}\left(1-\frac{\varepsilon^{2}}{6 r_{0}^{2}}\right)\left[\left(D_{2}^{1}-D_{1}^{2}\right)\left(r_{0}^{2}-5 x_{02}^{2}\right)-10\left(D_{1}^{3}-D_{3}^{1}\right) x_{02} x_{03}-10 x_{03}\left(x_{03} D_{1}^{2}-x_{01} D_{3}^{2}\right)\right. \\
& \left.-10 x_{02}\left(x_{01} D_{2}^{2}-x_{02} D_{1}^{2}\right)-10 x_{02}\left(x_{03} D_{1}^{3}-x_{01} D_{3}^{3}\right)\right]
\end{aligned}
$$




$$
\begin{aligned}
C_{32}= & \frac{3}{32} \pi \varepsilon^{2}\left(Q_{3} x_{01}-Q_{1} x_{03}\right)\left[5 \varepsilon^{2}\left(1-\frac{3 x_{03}^{2}}{r_{0}^{2}}\right)-4\left(r_{0}^{2}-5 x_{03}^{2}\right)\right]+\frac{15}{16} \pi \varepsilon^{2}\left(Q_{1} x_{02}-Q_{2} x_{01}\right)\left(4-\frac{3 \varepsilon^{2}}{r_{0}^{2}}\right) x_{02} x_{03} \\
& -\frac{3}{32} \pi \varepsilon^{4}\left(1-\frac{\varepsilon^{2}}{6 r_{0}^{2}}\right)\left[\left(D_{3}^{1}-D_{1}^{3}\right)\left(r_{0}^{2}-5 x_{03}^{2}\right)-10\left(D_{1}^{2}-D_{2}^{1}\right) x_{02} x_{03}-10 x_{02}\left(x_{02} D_{1}^{3}-x_{01} D_{2}^{3}\right)\right. \\
& \left.-10 x_{03}\left(x_{01} D_{3}^{3}-x_{03} D_{1}^{3}\right)-10 x_{03}\left(x_{02} D_{1}^{2}-x_{01} D_{2}^{2}\right)\right] \\
C_{31}= & \frac{3}{32} \pi \varepsilon^{2}\left(Q_{2} x_{03}-Q_{3} x_{02}\right)\left[5 \varepsilon^{2}\left(1-\frac{3 x_{03}^{2}}{r_{0}^{2}}\right)-4\left(r_{0}^{2}-5 x_{03}^{2}\right)\right]+\frac{15}{16} \pi \varepsilon^{2}\left(Q_{1} x_{02}-Q_{2} x_{01}\right)\left(4-\frac{3 \varepsilon^{2}}{r_{0}^{2}}\right) x_{01} x_{03} \\
& +\frac{3}{32} \pi \varepsilon^{4}\left(1-\frac{\varepsilon^{2}}{6 r_{0}^{2}}\right)\left[\left(D_{3}^{2}-D_{2}^{3}\right)\left(r_{0}^{2}-5 x_{03}^{2}\right)-10\left(D_{2}^{1}-D_{1}^{2}\right) x_{01} x_{03}-10 x_{01}\left(x_{01} D_{2}^{3}-x_{02} D_{1}^{3}\right)\right. \\
& \left.-10 x_{03}\left(x_{02} D_{3}^{3}-x_{03} D_{2}^{3}\right)-10 x_{03}\left(x_{01} D_{2}^{1}-x_{02} D_{1}^{1}\right)\right] \\
C_{13}= & \frac{3}{32} \pi \varepsilon^{2}\left(Q_{1} x_{02}-Q_{2} x_{01}\right)\left[5 \varepsilon^{2}\left(1-\frac{3 x_{01}^{2}}{r_{0}^{2}}\right)-4\left(r_{0}^{2}-5 x_{01}^{2}\right)\right]+\frac{15}{16} \pi \varepsilon^{2}\left(Q_{2} x_{03}-Q_{3} x_{02}\right)\left(4-\frac{3 \varepsilon^{2}}{r_{0}^{2}}\right) x_{01} x_{03} \\
& -\frac{3}{32} \pi \varepsilon^{4}\left(1-\frac{\varepsilon^{2}}{6 r_{0}^{2}}\right)\left[\left(D_{1}^{2}-D_{2}^{1}\right)\left(r_{0}^{2}-5 x_{01}^{2}\right)-10\left(D_{2}^{3}-D_{3}^{2}\right) x_{01} x_{03}-10 x_{03}\left(x_{03} D_{2}^{1}-x_{02} D_{3}^{1}\right)\right. \\
& \left.-10 x_{01}\left(x_{02} D_{1}^{1}-x_{01} D_{2}^{1}\right)-10 x_{01}\left(x_{03} D_{2}^{3}-x_{02} D_{3}^{3}\right)\right]
\end{aligned}
$$

where it is straightforward to verify the constraints (38)-(40). Finally, from the equality of the coefficients of the product terms $x_{1} x_{2} x_{3}$ in Equations (32) and (37) we obtain

$$
\begin{aligned}
C_{123}= & \frac{15}{16} \pi \varepsilon^{2}\left(4-3 \frac{\varepsilon^{2}}{r_{0}^{2}}\right)\left[\left(Q_{1} x_{02}-Q_{2} x_{01}\right) x_{01} x_{02}+\left(Q_{3} x_{01}-Q_{1} x_{03}\right) x_{01} x_{03}+\left(Q_{2} x_{03}-Q_{3} x_{02}\right) x_{02} x_{03}\right] \\
& -\frac{15}{16} \pi \varepsilon^{4}\left(1-\frac{\varepsilon^{2}}{6 r_{0}^{2}}\right)\left[\left(D_{2}^{1}-D_{1}^{2}\right) x_{01} x_{02}+\left(D_{3}^{2}-D_{2}^{3}\right) x_{02} x_{03}+\left(D_{1}^{3}-D_{3}^{1}\right) x_{01} x_{03}\right. \\
& +x_{01}\left(x_{01} D_{2}^{2}-x_{02} D_{1}^{2}+x_{03} D_{1}^{3}-x_{01} D_{3}^{3}\right)+x_{02}\left(x_{01} D_{2}^{1}-x_{02} D_{1}^{1}+x_{02} D_{3}^{3}-x_{03} D_{2}^{3}\right) \\
& \left.+x_{03}\left(x_{03} D_{1}^{1}-x_{01} D_{3}^{1}+x_{02} D_{3}^{2}-x_{03} D_{2}^{2}\right)\right] .
\end{aligned}
$$

The solution of the above nonlinear system of algebraic equations determines the position, the orientation and the size of the disk that supports the primary neuronal current. A series of numerical tests to obtain the solution of this system show that there exists a unique real solution.

\section{Acknowledgments}

The present work is part of the project "Functional Brain", which is implemented within the "ARISTEIA" Action of the "OPERATIONAL PROGRAMME EDUCATION AND LIFELONG LEARNING" and is cofunded by the European Social Fund (ESF) and National Resources.

\section{References}

[1] Dassios, G. and Kariotou, F. (2003) Magnetoencephalography in Ellipsoidal Geometry. Journal of Mathematical Physics, 44, 220-241. http://dx.doi.org/10.1063/1.1522135

[2] Hamalainen, M., Hari, R., Ilmoniemi, R.J., Knuutila, J. and Lounasmaa, O.V. (1993) Magnetoencephalography-Theory, Instrumentation, and Applications to Noninvasive Studies of the Working Human Brain. Reviewed Modern Physics, 65, 413-497.

[3] Malmivuo, J. and Plonsey, R. (1995) Bioelectromagnetism. Oxford University Press, New York.

[4] Helmholtz, H. (1853) Ueber einige Gesetze der Vertheilung elektrischer Strme in k orperlichen Leitern mit Anwen- 
dung auf die thierisch-elektrischen Versuche. Annalen der Physik und Chemie, 89, 211-233,353-377.

[5] Fokas, A.S. (2008) Electro-Magneto-Encephalography for the Three-Shell Model: Distributed Current in Arbitrary, Spherical and Ellipsoidal Geometries. Journal of the Royal Society Interface, 6, 479-488. http://dx.doi.org/10.1098/rsif.2008.0309

[6] Dassios, G. and Fokas, A.S. (2013) The Definitive Non Uniqueness Results for Deterministic EEG and MEG Data. Inverse Problems, 29, 1-10.

[7] Albanese, R. and Monk, P.B. (2006) The Inverse Source Problem for Maxwell’s Equations. Inverse Problems, 22, 1023-1035. http://dx.doi.org/10.1088/0266-5611/22/3/018

[8] Landau, L.D. and Lifshitz, E.M. (1960) Electrodynamics of Continuous Media. Pergamon Press, London.

[9] Plonsey, R. and Heppner, D.B. (1967) Considerations of Quasi-Stationarity in Electrophysiological Systems. Bulletin of Mathematical Biophysics, 29, 657-664. http://dx.doi.org/10.1007/BF02476917

[10] Sarvas, J. (1987) Basic Mathematical and Electromagnetic Concepts of the Biomagnetic Inverse Problems. Physics in Medicine and Biology, 32, 11-22. http://dx.doi.org/10.1088/0031-9155/32/1/004

[11] Geselowitz, D.B. (1970) On the Magnetic Field Generated outside an Inhomogeneous Volume Conductor by Internal Current Sources. IEEE Transactions in Biomagnetism, 6, 346-347. http://dx.doi.org/10.1109/TMAG.1970.1066765

[12] Dassios, G. (2009) Electric and Magnetic Activity of the Brain in Spherical and Ellipsoidal Geometry. In: Ammari, H., Ed., Mathematical Modeling in Biomedical Imaging, Mathematical Biosciences Subseries, Springer-Verlag, 183, 133202.

[13] Dassios, G. (2012) Ellipsoidal Harmonics. Theory and Applications. Cambridge University Press, Cambridge. http://dx.doi.org/10.1017/CBO9781139017749

[14] Dassios, G. and Fokas, A.S. (2009) Electro-Magneto-Encephalography and Fundamental Solutions. Quarterly of Applied Mathematics, 67, 771-780.

[15] Dassios, G. and Fokas, A.S. (2009) Electro-Magneto-Encephalography for the Three-Shell Model: Dipoles and Beyond for the Spherical Geometry. Inverse Problems, 25, 1-20.

[16] Brand, L. (1947) Vector and Tensor Analysis. John Wiley and Sons, New York.

[17] Morse, P.M. and Feshbach, H. (1953) Methods of Theoretical Physics. McGraw-Hill, New York. 


\section{Appendix}

The four basic integrals we need to calculate are

$$
\begin{gathered}
\boldsymbol{I}_{1}=\oint_{D_{\varepsilon}} \boldsymbol{r d s} \\
\boldsymbol{I}_{2}=\oint_{D_{\varepsilon}} \boldsymbol{r} \otimes \boldsymbol{r} \mathrm{d} s \\
\boldsymbol{I}_{3}=\oint_{D_{\varepsilon}} \boldsymbol{r} \otimes \boldsymbol{r} \otimes \boldsymbol{r d s} \\
\boldsymbol{I}_{4}=\oint_{D_{\varepsilon}} \boldsymbol{r} \otimes \boldsymbol{r} \otimes \boldsymbol{r} \otimes \boldsymbol{r d s} .
\end{gathered}
$$

Choosing $\boldsymbol{r}_{0}=r_{0} \hat{\boldsymbol{x}}_{3}$ and using cylindrical coordinates, so that

$$
\boldsymbol{r}=\left(\rho \cos \varphi, \rho \sin \varphi, r_{0}\right), \rho \in[0, \varepsilon], \varphi \in[0,2 \pi)
$$

we obtain

$$
\boldsymbol{I}_{1}=\int_{0}^{2 \pi} \int_{0}^{\varepsilon}\left(\rho \cos \varphi \hat{\boldsymbol{x}}_{1}+\rho \sin \varphi \hat{\boldsymbol{x}}_{2}+r_{0} \hat{\boldsymbol{X}}_{3}\right) \rho \mathrm{d} \rho \mathrm{d} \varphi=\pi \varepsilon^{2} r_{0} \hat{\boldsymbol{x}}_{3} .
$$

Similarly,

$$
\begin{aligned}
\boldsymbol{I}_{2} & =\int_{0}^{2 \pi} \int_{0}^{\varepsilon}\left(\rho \cos \varphi \hat{\boldsymbol{x}}_{1}+\rho \sin \varphi \hat{\boldsymbol{x}}_{2}+r_{0} \hat{\boldsymbol{x}}_{3}\right) \otimes\left(\rho \cos \varphi \hat{\boldsymbol{x}}_{1}+\rho \sin \varphi \hat{\boldsymbol{x}}_{2}+r_{0} \hat{\boldsymbol{x}}_{3}\right) \rho \mathrm{d} \rho \mathrm{d} \varphi \\
& =\int_{0}^{2 \pi} \int_{0}^{\varepsilon}\left(\rho^{2} \cos ^{2} \varphi \hat{\boldsymbol{x}}_{1} \otimes \hat{\boldsymbol{x}}_{1}+\rho^{2} \sin ^{2} \varphi \hat{\boldsymbol{x}}_{2} \otimes \hat{\boldsymbol{x}}_{2}+r_{0}^{2} \hat{\boldsymbol{x}}_{3} \otimes \hat{\boldsymbol{x}}_{3}\right) \rho \mathrm{d} \rho \mathrm{d} \varphi \\
& =\frac{\pi \varepsilon^{4}}{4}\left(\hat{\boldsymbol{x}}_{1} \otimes \hat{\boldsymbol{x}}_{1}+\hat{\boldsymbol{x}}_{2} \otimes \hat{\boldsymbol{x}}_{2}\right)+\pi \varepsilon^{2} r_{0}^{2} \hat{\boldsymbol{x}}_{3} \otimes \hat{\boldsymbol{x}}_{3} \\
& =\frac{\pi \varepsilon^{4}}{4} \tilde{\boldsymbol{I}}+\frac{\pi \varepsilon^{2}}{4}\left(4 r_{0}^{2}-\varepsilon^{2}\right) \hat{\boldsymbol{x}}_{3} \otimes \hat{\boldsymbol{x}}_{3}
\end{aligned}
$$

and

$$
\begin{aligned}
\boldsymbol{I}_{3}= & \int_{0}^{2 \pi} \int_{0}^{\varepsilon}\left(\rho \cos \varphi \hat{\boldsymbol{x}}_{1}+\rho \sin \varphi \hat{\boldsymbol{x}}_{2}+r_{0} \hat{\boldsymbol{x}}_{3}\right) \otimes\left(\rho \cos \varphi \hat{\boldsymbol{x}}_{1}+\rho \sin \varphi \hat{\boldsymbol{x}}_{2}+r_{0} \hat{\boldsymbol{x}}_{3}\right) \\
& \otimes\left(\rho \cos \varphi \hat{\boldsymbol{x}}_{1}+\rho \sin \varphi \hat{\boldsymbol{x}}_{2}+r_{0} \hat{\boldsymbol{x}}_{3}\right) \rho \mathrm{d} \rho \mathrm{d} \varphi \\
= & \frac{\varepsilon^{4} r_{0}}{4}\left(\hat{\boldsymbol{x}}_{2} \otimes \hat{\boldsymbol{x}}_{2} \otimes \hat{\boldsymbol{x}}_{3}+\hat{\boldsymbol{x}}_{2} \otimes \hat{\boldsymbol{x}}_{3} \otimes \hat{\boldsymbol{x}}_{2}+\hat{\boldsymbol{x}}_{3} \otimes \hat{\boldsymbol{x}}_{2} \otimes \hat{\boldsymbol{x}}_{2}\right) \int_{0}^{2 \pi} \sin ^{2} \varphi \mathrm{d} \varphi \\
& +\frac{\varepsilon^{4} r_{0}}{4}\left(\hat{\boldsymbol{x}}_{1} \otimes \hat{\boldsymbol{x}}_{1} \otimes \hat{\boldsymbol{x}}_{3}+\hat{\boldsymbol{x}}_{1} \otimes \hat{\boldsymbol{x}}_{3} \otimes \hat{\boldsymbol{x}}_{1}+\hat{\boldsymbol{x}}_{3} \otimes \hat{\boldsymbol{x}}_{1} \otimes \hat{\boldsymbol{x}}_{1}\right) \int_{0}^{2 \pi} \cos ^{2} \varphi \mathrm{d} \varphi \\
& +\frac{\varepsilon^{2} r_{0}^{3}}{2} \hat{\boldsymbol{x}}_{3} \otimes \hat{\boldsymbol{x}}_{3} \otimes \hat{\boldsymbol{x}}_{3} \int_{0}^{2 \pi} \mathrm{d} \varphi \\
= & \frac{\pi \varepsilon^{4} r_{0}}{4}\left[\left(\hat{\boldsymbol{x}}_{1} \otimes \hat{\boldsymbol{x}}_{1}+\hat{\boldsymbol{x}}_{2} \otimes \hat{\boldsymbol{x}}_{2}\right) \otimes \hat{\boldsymbol{x}}_{3}+\hat{\boldsymbol{x}}_{3} \otimes\left(\hat{\boldsymbol{x}}_{1} \otimes \hat{\boldsymbol{x}}_{1}+\hat{\boldsymbol{x}}_{2} \otimes \hat{\boldsymbol{x}}_{2}\right)\right. \\
& \left.+\hat{\boldsymbol{x}}_{1} \otimes \hat{\boldsymbol{x}}_{3} \otimes \hat{\boldsymbol{x}}_{1}+\hat{\boldsymbol{x}}_{2} \otimes \hat{\boldsymbol{x}}_{3} \otimes \hat{\boldsymbol{x}}_{2}\right]+\pi \varepsilon^{2} r_{0}^{3} \hat{\boldsymbol{x}}_{3} \otimes \hat{\boldsymbol{x}}_{3} \otimes \hat{\boldsymbol{x}}_{3} \\
= & \frac{\pi \varepsilon^{4} r_{0}}{4}\left[\hat{\boldsymbol{x}}_{3} \otimes \tilde{\boldsymbol{I}}+\tilde{\boldsymbol{I}} \otimes \hat{\boldsymbol{x}}_{3}+\hat{\boldsymbol{x}}_{1} \otimes \hat{\boldsymbol{x}}_{3} \otimes \hat{\boldsymbol{x}}_{1}+\hat{\boldsymbol{x}}_{2} \otimes \hat{\boldsymbol{x}}_{3} \otimes \hat{\boldsymbol{x}}_{2}\right] \\
& +\frac{\pi \varepsilon^{2} r_{0}}{2}\left(2 r_{0}^{2}-\varepsilon^{2}\right) \hat{\boldsymbol{x}}_{3} \otimes \hat{\boldsymbol{x}}_{3} \otimes \hat{\boldsymbol{x}}_{3} .
\end{aligned}
$$

Finally, for the tetradic integral [16] we obtain

$$
\begin{aligned}
\boldsymbol{I}_{4}= & \int_{0}^{2 \pi} \int_{0}^{\varepsilon}\left(\rho \cos \varphi \hat{\boldsymbol{x}}_{1}+\rho \sin \varphi \hat{\boldsymbol{x}}_{2}+r_{0} \hat{\boldsymbol{x}}_{3}\right) \otimes\left(\rho \cos \varphi \hat{\boldsymbol{x}}_{1}+\rho \sin \varphi \hat{\boldsymbol{x}}_{2}+r_{0} \hat{\boldsymbol{x}}_{3}\right) \\
& \otimes\left(\rho \cos \varphi \hat{\boldsymbol{x}}_{1}+\rho \sin \varphi \hat{\boldsymbol{x}}_{2}+r_{0} \hat{\boldsymbol{x}}_{3}\right) \otimes\left(\rho \cos \varphi \hat{\boldsymbol{x}}_{1}+\rho \sin \varphi \hat{\boldsymbol{x}}_{2}+r_{0} \hat{\boldsymbol{x}}_{3}\right) \rho \mathrm{d} \rho \mathrm{d} \varphi
\end{aligned}
$$


which involves $3^{4}=81$ tetrads. However, the only tetrads that are not multiplied by a vanishing factor are those that contain the following five integrals

$$
\begin{gathered}
\int_{0}^{2 \pi} \sin ^{2} \varphi \mathrm{d} \varphi=\int_{0}^{2 \pi} \cos ^{2} \varphi \mathrm{d} \varphi=\pi \\
\int_{0}^{2 \pi} \sin ^{4} \varphi \mathrm{d} \varphi=\int_{0}^{2 \pi} \cos ^{4} \varphi \mathrm{d} \varphi=\frac{3 \pi}{4} \\
\int_{0}^{2 \pi} \sin ^{2} \varphi \cos ^{2} \varphi \mathrm{d} \varphi=\frac{\pi}{4} .
\end{gathered}
$$

Therefore, we arrive at the following combination of the survived 21 tetrads

$$
\begin{aligned}
\boldsymbol{I}_{4}= & \frac{\pi \varepsilon^{6}}{24}\left[3 \hat{\boldsymbol{x}}_{1} \otimes \hat{\boldsymbol{x}}_{1} \otimes \hat{\boldsymbol{x}}_{1} \otimes \hat{\boldsymbol{x}}_{1}+3 \hat{\boldsymbol{x}}_{2} \otimes \hat{\boldsymbol{x}}_{2} \otimes \hat{\boldsymbol{x}}_{2} \otimes \hat{\boldsymbol{x}}_{2}+\hat{\boldsymbol{x}}_{1} \otimes \hat{\boldsymbol{x}}_{1} \otimes \hat{\boldsymbol{x}}_{2} \otimes \hat{\boldsymbol{x}}_{2}+\hat{\boldsymbol{x}}_{2} \otimes \hat{\boldsymbol{x}}_{2} \otimes \hat{\boldsymbol{x}}_{1} \otimes \hat{\boldsymbol{x}}_{1}\right. \\
& \left.+\hat{\boldsymbol{x}}_{1} \otimes \hat{\boldsymbol{x}}_{2} \otimes \hat{\boldsymbol{x}}_{1} \otimes \hat{\boldsymbol{x}}_{2}+\hat{\boldsymbol{x}}_{1} \otimes \hat{\boldsymbol{x}}_{2} \otimes \hat{\boldsymbol{x}}_{2} \otimes \hat{\boldsymbol{x}}_{1}+\hat{\boldsymbol{x}}_{2} \otimes \hat{\boldsymbol{x}}_{1} \otimes \hat{\boldsymbol{x}}_{2} \otimes \hat{\boldsymbol{x}}_{1}+\hat{\boldsymbol{x}}_{2} \otimes \hat{\boldsymbol{x}}_{1} \otimes \hat{\boldsymbol{x}}_{1} \otimes \hat{\boldsymbol{x}}_{2}\right] \\
& +\frac{\pi \varepsilon^{4} r_{0}^{2}}{4}\left[\hat{\boldsymbol{x}}_{1} \otimes \hat{\boldsymbol{x}}_{1} \otimes \hat{\boldsymbol{x}}_{3} \otimes \hat{\boldsymbol{x}}_{3}+\hat{\boldsymbol{x}}_{3} \otimes \hat{\boldsymbol{x}}_{3} \otimes \hat{\boldsymbol{x}}_{1} \otimes \hat{\boldsymbol{x}}_{1}+\hat{\boldsymbol{x}}_{2} \otimes \hat{\boldsymbol{x}}_{2} \otimes \hat{\boldsymbol{x}}_{3} \otimes \hat{\boldsymbol{x}}_{3}+\hat{\boldsymbol{x}}_{3} \otimes \hat{\boldsymbol{x}}_{3} \otimes \hat{\boldsymbol{x}}_{2} \otimes \hat{\boldsymbol{x}}_{2}\right. \\
& +\hat{\boldsymbol{x}}_{1} \otimes \hat{\boldsymbol{x}}_{3} \otimes \hat{\boldsymbol{x}}_{1} \otimes \hat{\boldsymbol{x}}_{3}+\hat{\boldsymbol{x}}_{1} \otimes \hat{\boldsymbol{x}}_{3} \otimes \hat{\boldsymbol{x}}_{3} \otimes \hat{\boldsymbol{x}}_{1}+\hat{\boldsymbol{x}}_{3} \otimes \hat{\boldsymbol{x}}_{1} \otimes \hat{\boldsymbol{x}}_{1} \otimes \hat{\boldsymbol{x}}_{3}+\hat{\boldsymbol{x}}_{3} \otimes \hat{\boldsymbol{x}}_{1} \otimes \hat{\boldsymbol{x}}_{3} \otimes \hat{\boldsymbol{x}}_{1} \\
& \left.+\hat{\boldsymbol{x}}_{2} \otimes \hat{\boldsymbol{x}}_{3} \otimes \hat{\boldsymbol{x}}_{2} \otimes \hat{\boldsymbol{x}}_{3}+\hat{\boldsymbol{x}}_{2} \otimes \hat{\boldsymbol{x}}_{3} \otimes \hat{\boldsymbol{x}}_{3} \otimes \hat{\boldsymbol{x}}_{2}+\hat{\boldsymbol{x}}_{3} \otimes \hat{\boldsymbol{x}}_{2} \otimes \hat{\boldsymbol{x}}_{2} \otimes \hat{\boldsymbol{x}}_{3}+\hat{\boldsymbol{x}}_{3} \otimes \hat{\boldsymbol{x}}_{2} \otimes \hat{\boldsymbol{x}}_{3} \otimes \hat{\boldsymbol{x}}_{2}\right] \\
& +\pi \varepsilon^{2} r_{0}^{4} \hat{\boldsymbol{x}}_{3} \otimes \hat{\boldsymbol{x}}_{3} \otimes \hat{\boldsymbol{x}}_{3} \otimes \hat{\boldsymbol{x}}_{3} .
\end{aligned}
$$

The integral $\boldsymbol{I}_{4}$ appears in Formula (32) in the form

$$
\oint_{D_{\varepsilon}} \boldsymbol{r}^{\prime} \otimes \boldsymbol{r}^{\prime} \otimes\left(\boldsymbol{r}^{\prime} \cdot \tilde{\boldsymbol{D}} \times \boldsymbol{r}^{\prime}\right) \mathrm{d} s\left(\boldsymbol{r}^{\prime}\right)
$$

which, in view of the condition (14), implies that whenever the third vector of a tetrad is the base vector $\hat{\boldsymbol{x}}_{3}$ the corresponding term vanishes. Hence,

$$
\begin{aligned}
\oint_{D_{\varepsilon}} \boldsymbol{r}^{\prime} \otimes \boldsymbol{r}^{\prime} \otimes\left(\boldsymbol{r}^{\prime} \cdot \tilde{\boldsymbol{D}} \times \boldsymbol{r}^{\prime}\right) \mathrm{d} s\left(\boldsymbol{r}^{\prime}\right) \\
=\frac{\pi \varepsilon^{6}}{24}\left[\left(3 \hat{\boldsymbol{x}}_{1} \otimes \hat{\boldsymbol{x}}_{1}+\hat{\boldsymbol{x}}_{2} \otimes \hat{\boldsymbol{x}}_{2}\right) \otimes\left(\hat{\boldsymbol{x}}_{1} \cdot \tilde{\boldsymbol{D}} \times \hat{\boldsymbol{x}}_{1}\right)+\left(\hat{\boldsymbol{x}}_{1} \otimes \hat{\boldsymbol{x}}_{1}+3 \hat{\boldsymbol{x}}_{2} \otimes \hat{\boldsymbol{x}}_{2}\right)\right. \\
\left.\quad \otimes\left(\hat{\boldsymbol{x}}_{2} \cdot \tilde{\boldsymbol{D}} \times \hat{\boldsymbol{x}}_{2}\right)+\left(\hat{\boldsymbol{x}}_{1} \otimes \hat{\boldsymbol{x}}_{2}+\hat{\boldsymbol{x}}_{2} \otimes \hat{\boldsymbol{x}}_{1}\right) \otimes\left(\hat{\boldsymbol{x}}_{1} \cdot \tilde{\boldsymbol{D}} \times \hat{\boldsymbol{x}}_{2}+\hat{\boldsymbol{x}}_{2} \cdot \tilde{\boldsymbol{D}} \times \hat{\boldsymbol{x}}_{1}\right)\right] \\
\quad+\frac{\pi \varepsilon^{4} r_{0}^{2}}{4}\left[\hat{\boldsymbol{x}}_{3} \otimes \hat{\boldsymbol{x}}_{3} \otimes\left(\hat{\boldsymbol{x}}_{1} \cdot \tilde{\boldsymbol{D}} \times \hat{\boldsymbol{x}}_{1}+\hat{\boldsymbol{x}}_{2} \cdot \tilde{\boldsymbol{D}} \times \hat{\boldsymbol{x}}_{2}\right)+\left(\hat{\boldsymbol{x}}_{1} \otimes \hat{\boldsymbol{x}}_{3}+\hat{\boldsymbol{x}}_{3} \otimes \hat{\boldsymbol{x}}_{1}\right)\right. \\
\left.\quad \otimes\left(\hat{\boldsymbol{x}}_{1} \cdot \tilde{\boldsymbol{D}} \times \hat{\boldsymbol{x}}_{3}\right)+\left(\hat{\boldsymbol{x}}_{2} \otimes \hat{\boldsymbol{x}}_{3}+\hat{\boldsymbol{x}}_{3} \otimes \hat{\boldsymbol{x}}_{2}\right) \otimes\left(\hat{\boldsymbol{x}}_{2} \cdot \tilde{\boldsymbol{D}} \times \hat{\boldsymbol{x}}_{3}\right)\right] .
\end{aligned}
$$

Substituting $\boldsymbol{I}_{3}$ and $\boldsymbol{I}_{4}$ in the integral expression of $H_{3}$, as it is given in (32) and performing the indicated algebra, we arrive at the expression

$$
\begin{aligned}
H_{3}(\boldsymbol{r})= & \frac{15 \pi \varepsilon^{2} r_{0}}{8}\left[\left(\frac{3 \varepsilon^{2}}{4}-r_{0}^{2}\right)\left(\boldsymbol{r} \cdot \hat{\boldsymbol{x}}_{3}\right)^{2}+r^{2}\left(\frac{r_{0}^{2}}{5}-\frac{\varepsilon^{2}}{4}\right)\right]\left(\boldsymbol{r} \cdot \hat{\boldsymbol{x}}_{3} \times \boldsymbol{Q}\right) \\
& -\frac{5 \pi \varepsilon^{6}}{64}\left[\left(\boldsymbol{r} \cdot \hat{\boldsymbol{x}}_{1}\right)^{2}\left(\tilde{\boldsymbol{I}}+2 \hat{\boldsymbol{x}}_{1} \otimes \hat{\boldsymbol{x}}_{1}\right)+\left(\boldsymbol{r} \cdot \hat{\boldsymbol{x}}_{2}\right)^{2}\left(\tilde{\boldsymbol{I}}+2 \hat{\boldsymbol{x}}_{2} \otimes \hat{\boldsymbol{x}}_{2}\right)\right. \\
& \left.+2\left(\boldsymbol{r} \cdot \hat{\boldsymbol{x}}_{1}\right)\left(\boldsymbol{r} \cdot \hat{\boldsymbol{x}}_{2}\right)\left(\hat{\boldsymbol{x}}_{1} \otimes \hat{\boldsymbol{x}}_{2}+\hat{\boldsymbol{x}}_{2} \otimes \hat{\boldsymbol{x}}_{1}\right)\right]: \tilde{\boldsymbol{D}} \times \boldsymbol{r} \\
& -\frac{15 \pi \varepsilon^{4} r_{0}^{2}}{32}\left(\boldsymbol{r} \cdot \hat{\boldsymbol{x}}_{3}\right)\left[2\left(\boldsymbol{r} \cdot \hat{\boldsymbol{x}}_{1}\right) \hat{\boldsymbol{x}}_{3} \otimes \hat{\boldsymbol{x}}_{1}+2\left(\boldsymbol{r} \cdot \hat{\boldsymbol{x}}_{2}\right) \hat{\boldsymbol{x}}_{3} \otimes \hat{\boldsymbol{x}}_{2}+\left(\boldsymbol{r} \cdot \hat{\boldsymbol{x}}_{3}\right) \tilde{\boldsymbol{I}}\right]: \tilde{\boldsymbol{D}} \times \boldsymbol{r} \\
& +\frac{\pi \varepsilon^{4} r^{2}}{32}\left[2 \varepsilon^{2}+3 r_{0}^{2}\right](\boldsymbol{r} \cdot \boldsymbol{d}) .
\end{aligned}
$$

Further manipulations show that 


$$
\begin{gathered}
\left(\boldsymbol{r} \cdot \hat{\boldsymbol{x}}_{1}\right)^{2}+\left(\boldsymbol{r} \cdot \hat{\boldsymbol{x}}_{2}\right)^{2}=\boldsymbol{r} \otimes \boldsymbol{r}:\left(\tilde{\boldsymbol{I}}-\hat{\boldsymbol{x}}_{3} \otimes \hat{\boldsymbol{x}}_{3}\right) \\
{\left[\left(\boldsymbol{r} \cdot \hat{\boldsymbol{x}}_{1}\right)^{2} \hat{\boldsymbol{x}}_{1} \otimes \hat{\boldsymbol{x}}_{1}+\left(\boldsymbol{r} \cdot \hat{\boldsymbol{x}}_{2}\right)^{2} \hat{\boldsymbol{x}}_{2} \otimes \hat{\boldsymbol{x}}_{2}+\left(\boldsymbol{r} \cdot \hat{\boldsymbol{x}}_{1}\right)\left(\boldsymbol{r} \cdot \hat{\boldsymbol{x}}_{2}\right)\left(\hat{\boldsymbol{x}}_{1} \otimes \hat{\boldsymbol{x}}_{2}+\hat{\boldsymbol{x}}_{2} \otimes \hat{\boldsymbol{x}}_{1}\right)\right]: \tilde{\boldsymbol{D}} \times \boldsymbol{r}=\boldsymbol{r} \otimes \boldsymbol{r}:\left(\boldsymbol{r} \cdot \tilde{\boldsymbol{D}} \times \hat{\boldsymbol{x}}_{3}\right) \otimes \hat{\boldsymbol{x}}_{3}} \\
{\left[\left(\boldsymbol{r} \cdot \hat{\boldsymbol{x}}_{1}\right) \hat{\boldsymbol{x}}_{3} \otimes \hat{\boldsymbol{x}}_{1}+\left(\boldsymbol{r} \cdot \hat{\boldsymbol{x}}_{2}\right) \hat{\boldsymbol{x}}_{3} \otimes \hat{\boldsymbol{x}}_{2}\right]: \tilde{\boldsymbol{D}} \times \boldsymbol{r}=\hat{\boldsymbol{x}}_{3} \otimes \boldsymbol{r}: \tilde{\boldsymbol{D}} \times \boldsymbol{r} .}
\end{gathered}
$$

In view of these identities the expression for $\mathrm{H}_{3}$ is rewritten as

$$
\begin{aligned}
H_{3}(\boldsymbol{r})= & \frac{3 \pi \varepsilon^{2} r_{0}^{3}}{32 r_{0}^{2}}\left[r^{2}\left(4 r_{0}^{2}-5 \varepsilon^{2}\right)-5\left(\boldsymbol{r} \cdot \hat{\boldsymbol{x}}_{3}\right)^{2}\left(4 r_{0}^{2}-3 \varepsilon^{2}\right)\right]\left(\boldsymbol{r} \cdot \hat{\boldsymbol{x}}_{3} \times \boldsymbol{Q}\right) \\
& +\frac{5 \pi \varepsilon^{4}}{32}\left(r_{0}^{2}-\frac{\varepsilon^{2}}{6}\right)\left[\frac{3}{5} r^{2} \tilde{\boldsymbol{I}}-3\left(\boldsymbol{r} \cdot \hat{\boldsymbol{x}}_{3}\right)^{2} \tilde{\boldsymbol{I}}-6\left(\boldsymbol{r} \cdot \hat{\boldsymbol{x}}_{3}\right) \hat{\boldsymbol{x}}_{3} \otimes \boldsymbol{r}\right]: \tilde{\boldsymbol{D}} \times \boldsymbol{r} .
\end{aligned}
$$

The above intermediate calculations are used to derive Formulae (30), (31) and (32), where the special direction $\hat{\boldsymbol{x}}_{3}$ is replaced by the arbitrary direction $\hat{\boldsymbol{r}}_{0}$. 
Scientific Research Publishing (SCIRP) is one of the largest Open Access journal publishers. It is currently publishing more than 200 open access, online, peer-reviewed journals covering a wide range of academic disciplines. SCIRP serves the worldwide academic communities and contributes to the progress and application of science with its publication.

Other selected journals from SCIRP are listed as below. Submit your manuscript to us via either submit@scirp.org or Online Submission Portal.
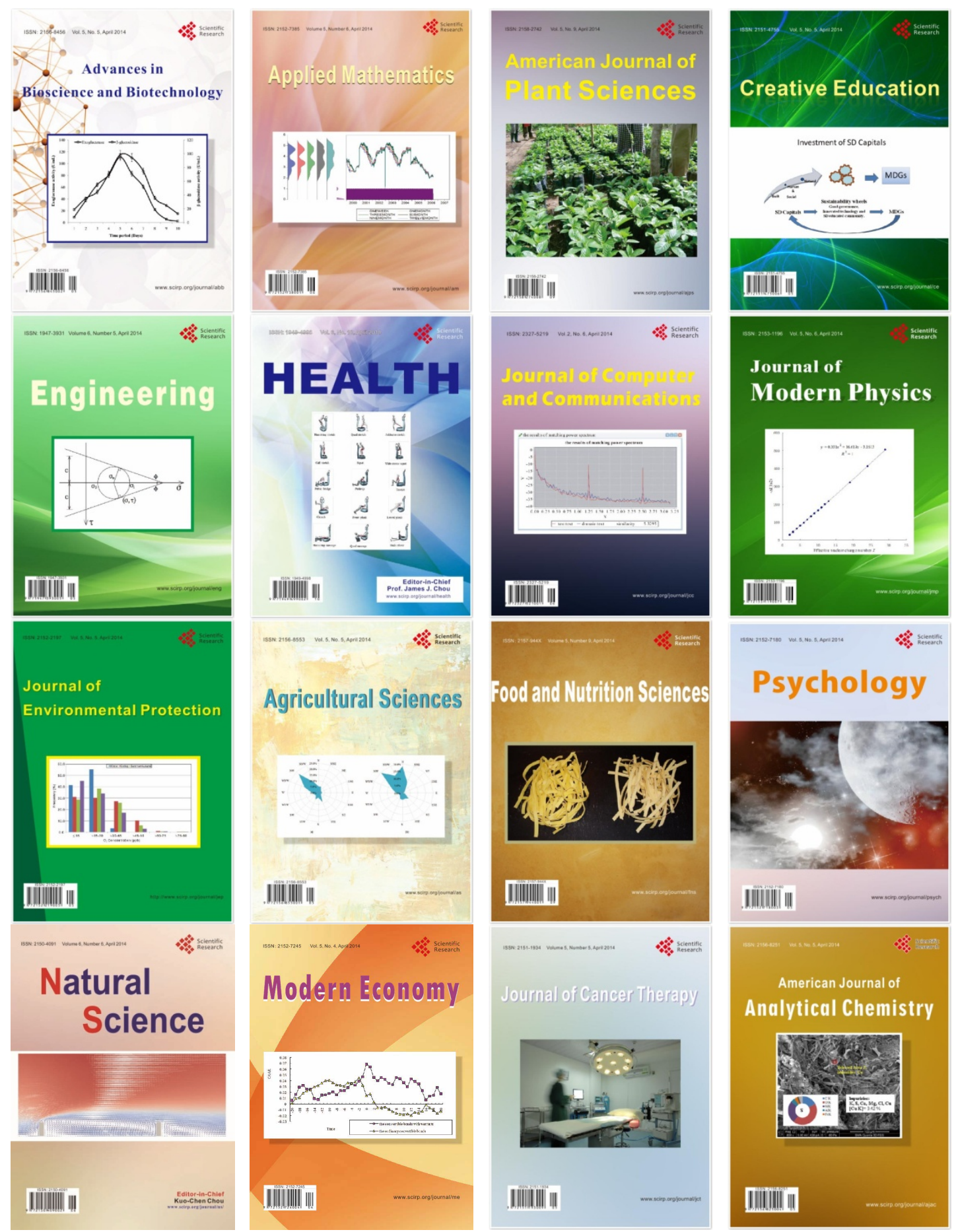